\title{
High level of initial default among smear positive pulmonary tuberculosis in eThekwini health district, KwaZulu-Natal
}

\author{
Lindiwe P Cele ${ }^{a}$, Stephen Knight ${ }^{b *}$, Elize Webb ${ }^{c}$, Khin Tint ${ }^{d}$ and Thembelihle Dlungwane ${ }^{b}$ \\ aNational Health Laboratory Services, Durban, South Africa \\ ${ }^{\circ}$ School of Nursing and Public Health, University of KwaZulu-Natal, Durban, South Africa \\ 'School of Health Systems and Public Health, SHSPH, University of Pretoria, Pretoria, South Africa \\ ${ }^{d}$ SAFELTP NICD, University of Pretoria, Pretoria, South Africa \\ *Corresponding author, email: knights@ukzn.ac.za
}

In this study, initial tuberculosis (TB) default was measured in clinics in a district with a high incidence of TB. A retrospective review of TB case identification registers in 2007 found 4049 (9.0\%) new smear positive pulmonary tuberculosis (PTB) cases from 44987 suspects. There were 725 (17.9\%) initial defaulters. Fifty-three percent of those traced had already died. TB is the leading cause of death in South Africa due to an infectious disease, and the high level of initial defaults in PTB is one of the "unresolved issues" in TB control programmes that needs to be addressed.

Keywords: initial default, KwaZulu-Natal, tuberculosis, turnaround time for sputum smear microscopy

\begin{abstract}
Introduction
South Africa (SA) is experiencing an unprecedented human immunodeficiency virus associated tuberculosis epidemic (HIVTB). ${ }^{1}$ The incidence of TB in SA increased from 305 per 100000 population in 1997 to 799 per 100000 in 2008. ${ }^{2}$ In KwaZulu-Natal (KZN) province the incidence of TB was 1076 per 100000 population and $80 \%$ of TB patients were co-infected with HIV. ${ }^{2}$ In $2009,55 \%$ of those who commenced TB treatment in KZN were cured, compared to $68 \%$ nationally. ${ }^{1}$ These TB outcomes are below the World Health Organisation (WHO) target for TB control of curing $85 \%$ of newly detected cases. Early diagnosis and prompt effective treatment is needed to reduce the TB burden and interrupt the TB transmission cycle. ${ }^{3}$
\end{abstract}

An initial defaulter is someone who has been diagnosed with sputum-confirmed PTB, but has no record of having started treatment so remains an infection risk for others. ${ }^{4}$ Initial default (ID) is one of the 'unresolved issues' in TB control and a problem to be addressed by health services at all levels. ${ }^{4}$

Reports of ID range from $4.5 \%{ }^{5}$ to $38 \%{ }^{6}$ and Mandal et al. cite more than 20 reported studies on ID in as many years. ${ }^{7}$ The level of ID varied widely depending on whether it was measured in urban or rural areas, in clinics or community-based settings, and whether the record of ID was electronically verified or the data manually extracted from TB registers. ${ }^{7}$ In the Western Cape of SA, ID was between $14 \%$ and $26 \%$ depending on whether the diagnosis of TB was based on a single or two sputum specimens. ${ }^{8,9}$ In KZN, $16 \%$ of PTB cases had not commenced treatment. ${ }^{10}$ Initial default is a substantial problem, but standardised measurement, reporting and its management remain a challenge for TB control.

The reasons given for ID include patient factors, such as ignorance, fear of stigmatisation, cost of returning to collect results and losing employment. Health service barriers that increase ID include inaccessible clinics with unfriendly staff. ${ }^{4,7,9}$
Managing ID raises challenges in TB control including how to identify these patients, trace them and get them on to treatment as soon as possible; how to register and report on these cases as part of the case-finding component of TB control; and, whether to include these initial defaulters in the cohort analysis of treatment outcomes. In HIV-TB, ID may be higher due to early death occurring between the diagnosis and starting treatment and delays in seeking care because of HIV-TB stigma. ${ }^{4}$

The aim of this study was to assess the frequency of ID in patients with sputum-confirmed pulmonary TB in eThekwini health district of KZN in 2007.

\section{Methods \\ Design \& setting}

A descriptive, cross-sectional study was used to assess the level of ID in 44987 pulmonary TB suspects attending 35 high TB burden clinics (that notified more than 250TB cases) in eThekwini health district in 2007.

\section{Definitions}

Initial defaulter - Patient with PTB without a record of having commenced treatment as reported in the case identification register or in the clinic-based TB treatment register.

Sputum-confirmed pulmonary TB: A diagnosis of PTB was made when a patient with suspected PTB had a single positive sputum smear.

\section{Measurements}

A list of PTB cases, their age, sex, subdistrict, the date TB treatment was commenced and counter-checked with the TB treatment register in each clinic was created manually and entered into a Microsoft Excel database. Output measures included the proportion of pulmonary TB suspects without confirmed diagnoses and the proportion of initial defaulters stratified by sex and age and were calculated using Microsoft Excel. 


\section{Ethics}

Ethical approval was obtained from the University of Pretoria's Research Ethics Committee (169/2008) and gate-keeper permission from the Department of Health.

\section{Results}

Of the 44987 pulmonary TB suspects registered at the highburden TB facilities in 2007, 4049 (9.0\%) had sputum-confirmed pulmonary TB. Their mean age was 34 years (standard deviation: 11.8 years). Their age ranged from 4 to 83 years. Females aged 15 to 29 years and males aged 30 to 39 years had significantly more PTB than other age groups $(p<0.001)$.

Only 977 (2.2\%) of PTB suspects had no record of laboratory results, of which 515 (53\%) had an inadequate specimen. In 298 (31\%) cases, the specimen was not brought to the clinic by the patient, in $18(2 \%)$ the specimen was not received by the laboratory, and in 146 (15\%) no reason was given for the lack of available result.

Initial default occurred in 725 (17.9\%; 95\% confidence interval (CI): 16.7 - 19.1) PTB cases (Figure 1). The largest proportion $(36.1 \%, 95 \% \mathrm{Cl}: 32.0$ - 39.9) was older than 40 years and $26.1 \%$ ( $95 \%$ Cl: 22.0 - 30.0) were between 30 to 34 years. There was no significant difference in ID between males (18.5\%; Cl: 16.8 - 20.3) and females (17.3\%; $95 \% \mathrm{Cl}: 15.6$ - 19.1) ( $p=0.48)$. The frequency of ID was not uniform in all subdistricts. It was $12.2 \%$ in the northern subdistrict, $27.2 \%$ in the southern subdistrict, and $13.6 \%$ in the western subdistrict.

Based on the address given by the patient and the availability of tracing teams, only $114(16 \%)$ of initial defaulters were traced, and $60(53 \%)$ of these had died before the commencement of TB treatment.

The turnaround time for sputum smear microscopy for 1706 (42\%) of the specimens was within the recommended 48-hour time period. ${ }^{11}$ Another 1309 (32.6\%) specimens took between

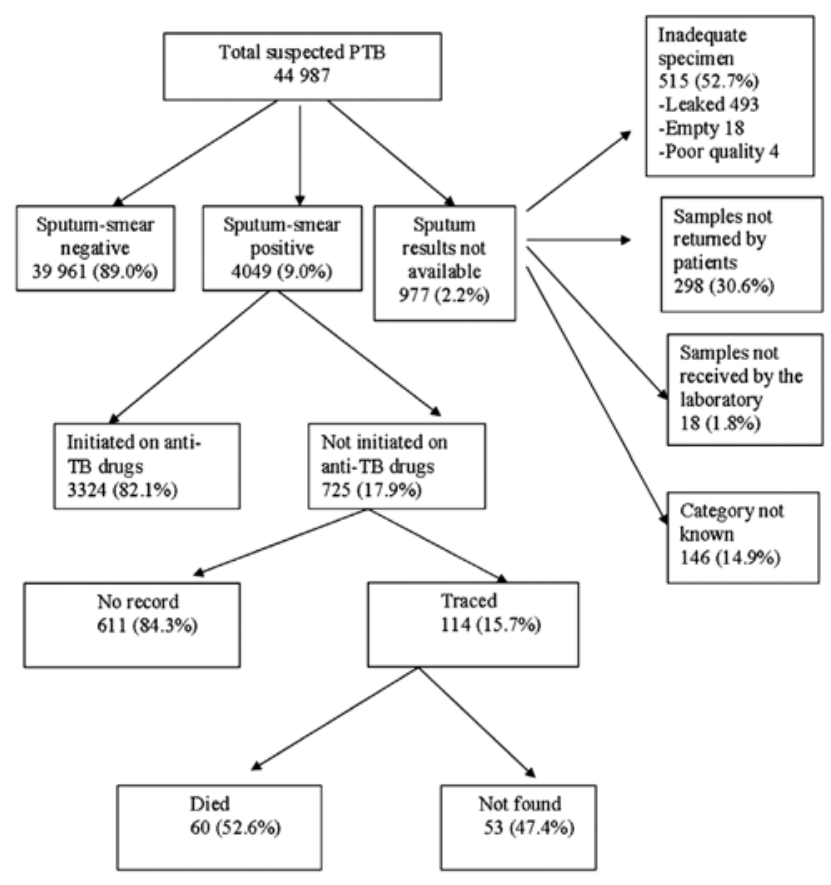

Figure 1: Pretreatment outcomes of tuberculosis suspects in high TB burden clinics in eThekwini health district. two to six days ( 120 hours) from the time of collection for the clinics to receive the result.

\section{Discussion}

An unacceptably high level of ID (18\%) was observed in this district, which was in the midrange of ID (4.5\% to $39 \%)$ reported from some other high TB burden countries. ${ }^{4-10}$ As these initial defaulters are not recorded in TB treatment registers and, therefore, not included in TB treatment outcome statistics, the actual number of true TB treatment defaulters would be higher than normally indicated by national TB control programme statistics. Initial default of 2-5\% was reported in KZN in 2013 by clinic-based passive surveillance. ${ }^{12}$ This study of 725 initial PTB defaulters involved a bigger sample than most reported studies, which ranged from 23 initial defaulters to 1453 in a state wide study from India. ${ }^{5}$

The definition of ID in reported studies is variable. Not all studies, including our study, used a standard definition of bacteriologically confirmed PTB. ${ }^{11}$ Early deaths in PTB cases are invariably reported as 'initial defaulters'. The high proportion of deaths in ID is a concern, suggesting advanced disease, inadequate care or early mortality among those HIV-TB coinfected. Our study was conducted early in the antiretroviral therapy roll-out phase of the HIV-TB epidemic in KZN. The case-fatality in eThekwini in 2014 has dropped to $4 \%$ but is still inexplicably high in some health districts in the province. ${ }^{12}$ The age and sex distribution of sputum-confirmed TB cases corresponded with other reported studies where younger women and older men bear the brunt of the HIV-associated TB burden.

With good case detection about $10 \%$ of sputum samples collected from PTB suspects should be sputum-confirmed. Case detection in our study (9.0\%) was comparable to studies in Ghana $(9.9 \%){ }^{13}$ but lower than Pakistan $(26 \%)^{14}$ and the Western Cape (14\% to $18 \%$ ), where auramine staining is used. ${ }^{8,9}$ Using more sensitive diagnostic tests would increase the diagnostic yield, but requiring two sputum-confirmed tests may decrease it. In this study, Ziehl-Neelsen staining was used. All laboratories in the district now use fluorescing auramine stain and Xpert ${ }^{\circ}$ MTB/ RIF tests. Even when more efficient diagnostics are available, patients with suspected PTB have to return later to obtain their results. ${ }^{15}$ The turn-around-time (79\% were less than 48 hours) has been improved with the expansion of rapid diagnostic test services and introduction of 'short-message-service' (SMS) machines in clinics to receive results rapidly from diagnostic centres..$^{12}$

Collecting sputum requires attention to technical detail from health care workers, otherwise the specimens arriving at the laboratory cannot be assessed. Although only a small proportion of the total specimens (2.2\%) were not suitable for examination this involved nearly 1000 specimens in a year from these clinics.

Only a small proportion of confirmed initial defaulters were traceable mainly due to resource constraints, but the lack of address documentation were also highlighted. In Pakistan, 'untraceable patients' were factors for high ID. ${ }^{13}$ TB tracing was often difficult in this district as many patients were of a migrant population and provide incorrect residential addresses. ${ }^{16}$ Similarly, in Cape Town, patient address-related problems were reported in $50 \%$ of ID cases. ${ }^{8}$ Currently there are many (20) 'outreach teams' in this district and most patients are contactable by cellular phone. 


\section{Limitations of the study}

This retrospective study used unverified data obtained manually from the facility, paper-based TB case identification and TB treatment registers. Further overestimation of ID was likely due to inadequate reporting of referrals, patients obtaining treatment from another clinic and patient registration challenges.

Data was collected at the height of the HIV-TB epidemic, when deaths and stigma were common and could have resulted in more ID. Tracing of initial defaulters was limited and the reasons for ID were not ascertained. Using only high-burden TB clinics may have resulted in an overestimate of the frequency of ID.

\section{Conclusion}

Initial default in sputum-confirmed PTB was unacceptably high in this urban district in KwaZulu-Natal. Despite the challenges identified with measuring and reporting ID, active management should lead to initial default being 'resolved'. Initial default is a preventable phenomenon which is critical to TB control at this facility, as well as on a national and global level.

\section{Recommendations}

The detection and confirmation of PTB requires a wellfunctioning health system and comprehensive inter-sectoral plan to facilitate access to diagnostic services and ensure that those diagnosed do indeed initiate TB treatment early and complete their full course of TB treatment. This plan should enable tracing of all defaulters and through pretreatment counselling promote an understanding of the importance of seeking early healthcare and an awareness of the implications of defaulting from TB treatment.

A stepwise approach to the challenges of initial defaulters should be adopted. Initial defaulters should be included in the TB treatment register and in the cohort analysis of treatment outcomes to avoid overestimating successful TB outcomes. Including initial defaulters demonstrates a proper understanding of the 'cohort' approach - being responsible for all cases that are present in the communities served. Recognising ID provides an opportunity to identify challenges to be addressed to improve the quality of access to patient care, which should lead to better TB control and improvements in mortality, prevalence and reduction of transmission of infection.

Further studies to explore the frequency and reasons for ID and, in particular, reasons for death before treatment initiation are needed.

\section{References}

1. South African National Aids Council. National Strategic on HIV, STIs AND TB 2012-2016. Pretoria: Department of National Health; 2011 [cited 2015 Mar 27]. Available from: http://www.sanac.org.za.

2. Day C, Grey A. Health and related indicators. In: Harrison S, Bhana R Ntuli A, editors. South African Health Review 2007. Durban: Health Systems Trust; 2007 [cited 2014 Jul]. Available from: http://www.hst. org.za/publications/711.

3. World Health Organization. Global Tuberculosis Report 2012 [cited 2014 Sep 10]. Available from: www.who.int/tb.

4. Harries $A D$, Rusen ID, Chiang $C Y$, et al. Registering initial defaulters and reporting on their treatment outcome. Int J Tuberc Lung Dis. 2009;13(7):801-3.

5. Sai Babu B, Satyanarayana AV, Venkateshwaralu G, et al. Initia default among diagnosed sputum smear-positive pulmonary tuberculosis patients in Andhra Pradesh, India. Int J Tuberc Lung Dis. 2008;12:1055-8.

6. Afutu FK, Zachariah R, Hinderaker SG, et al. High initial default in patients with smear-positive pulmonary tuberculosis at a regional hospital in Accra, Ghana. Trans R Soc Trop Med Hyg. 2012;106:511-3.

7. Mandal A, Basu M, Das $P$, et al. Magnitude and reasons of initial default among new sputum positive cases of pulmonary tuberculosis under RNTCP in a district of West Bengal, India. South East Asia J Public Health. 2014;4(1):41-7.

8. Botha $E$, Den Boon S, Verver S, et al. Initial default from tuberculosis treatment: how often does it happen and what are the reasons? Int Tuberc Lung Dis. 2008;12:820-3.

9. Botha $E$, den Boon S, Lawrence K-A, et al. From suspect to patient: tuberculosis diagnosis and treatment initiation in health facilities in South Africa. Int J Tuberc Lung Dis. 2008;12:936-41.

10. Loveday $M$, Thomson $L$, Chopra $M$, et al. A health systems assessment of the KwaZulu-Natal tuberculosis programme in the context of increasing drug resistance. Int J Tuberc Lung Dis. 2008;12(9):1042-7.

11. Department of Health. Diagnosis and management of TB: A manual for health care providers. 2003 [cited 2014 Apr 10]. Available from: www.doh.gov.za.

12. KwaZulu-Natal Health Department. 2013/14 Annual Report [cited 2015 May 15]. Available from: http://www.kznhealth.gov.za/ AnnualReport/2013_2014.pdf.

13. Afutu FK, Zachariah R, Hinderaker SG, et al. High initial default in patients with smear-positive pulmonary tuberculosis at a regional hospital in Accra, Ghana. Trans R Soc Trop Med Hyg. 2012;106:511-3.

14. Rao NA, Saleem M, Anwer T. Magnitude of initial default in pulmonary tuberculosis. J Pak Med Assoc. 2009;51:223-5.

15. Mntambo M, Mbatha M, Montague C. Feasibility and acceptability of implementing Xpert MTB/RIF in a public healthcare facility in rural KwaZulu-Natal. 2015. CAPRISA, Durban, South Africa.

16. Rajagopaul A, Kistnasamy EJ, Reddy P. Predictors of tuberculosis treatment defaulting in informal dwellers within the eThekwin Municipality, KwaZulu-Natal. South Afr J Epidemiol Infect. 2014;29(1):27-32. ISSN 1015-8782. 